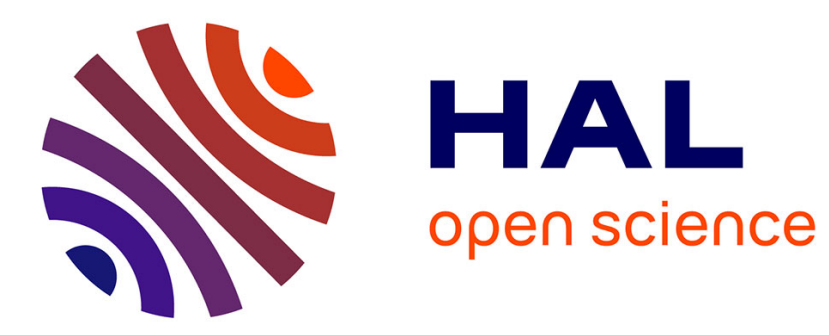

\title{
Violences ordinaires, violences enracinées, violences matricielles
}

Pierre Janin, Alain Marie

\section{To cite this version:}

Pierre Janin, Alain Marie. Violences ordinaires, violences enracinées, violences matricielles: Introduction au dossier. Politique africaine, 2003, 91, pp.5-12. ird-00275458

\section{HAL Id: ird-00275458 \\ https://hal.ird.fr/ird-00275458}

Submitted on 23 Apr 2008

HAL is a multi-disciplinary open access archive for the deposit and dissemination of scientific research documents, whether they are published or not. The documents may come from teaching and research institutions in France or abroad, or from public or private research centers.
L'archive ouverte pluridisciplinaire HAL, est destinée au dépôt et à la diffusion de documents scientifiques de niveau recherche, publiés ou non, émanant des établissements d'enseignement et de recherche français ou étrangers, des laboratoires publics ou privés. 


\title{
Violences ordinaires, violences enracinées, violences matricielles
}

\author{
par Pierre Janin et Alain Marie \\ Introduction au thème
}

La violence est un sujet brûlant, a fortiori dès lors que l'on traite de l'Afrique à propos de laquelle, selon les mouvements de balancier de l'africanisme autorisé, il convient tantôt d'être Cassandre (l'«afro-pessimisme » des amis consternés de l'Afrique), tantôt d'être Pangloss (l' « afro-optimisme » des amis encourageants de l'Afrique). Mais, entre les guerres «tribales », «ethniques », « civiles » ou même « lignagères », d'une part, et l'Afrique de la convivialité, de la solidarité, de l'ingéniosité des pauvres et des miracles quotidiens de l'économie populaire, d'autre part, faudrait-il donc choisir ? Et au nom de quelle position d'extériorité en surplomb ? $\mathrm{Ne}$ convient-il pas au contraire,plus modestement, d'observer et d'écouter les Africains eux-mêmes et de prendre acte de ce que, sur tous ces thèmes, ils ont, eux, une position beaucoup plus réaliste, donnant toute sa place à la pensée de l'ambivalence et au juste refus des alternatives abusivement simplificatrices?

Ainsi de la violence (la coercition morale ou physique « légitimée » par le droit du plus fort) dont on pourrait dire qu'en Afrique elle est la forme et l'instrument privilégiés de l'exercice du pouvoir institué et, par conséquent,de la lutte pour sa conquête et son contrôle, tandis qu'en face, « en bas ", le peuple des villages et des villes, culturellement enfermé dans des traditions de respect révérencieux à l'égard de l'autorité sous toutes ses formes, n'aurait d'autre issue que la soumission fataliste, le consentement stipendié, les micro stratégies de l'entrisme à petits pas, les «tactiques » de résistance passive ou indirecte, la débrouillardise « informelle » ou les soudaines poussées du désespoir révolté, aussitôt réprimées par le pouvoir ou bien dévoyées par des entrepreneurs politiques plus chefs de guerre ou de faction que leaders populistes...

\section{Violences ordinaires : violences enracinées}

Dès son numéro inaugural, Politique africaine ${ }^{1}$ invitait, contre la tendance à privilégier la scène étatique, à comprendre « la politique en Afrique noire » du point de vue d'une anthropologie du « politique par le bas ${ }^{2} »$. En s'inspirant de Foucault, il s'agissait de privilégier l'analyse des « dispositifs » qui « fonctionnent en sous-main », parce qu'ils sont immergés dans l'épaisseur des rapports sociaux ordinaires où ils produisent de la discipline et de la surveillance,contribuant ainsi à une «microphysique du pouvoir » dont on peut dire, pour le propos du présent dossier, qu'elle est indissociablement une microphysique de la violence », si l'on

\footnotetext{
${ }^{1}$ Politique africaine, «La politique en Afrique noire : le haut et le bas », $\mathrm{n}^{\circ} 1$, janvier 1981.

${ }^{2}$ 2. J.-F. Bayart, « Le politique par le bas en Afrique noire. Questions de méthode », ibid., pp. 53-82.
} 
considère que « là où il y a du pouvoir, il y a de la résistance » (Foucault), donc de la violence du pouvoir contre la violence de résistance.

Et en effet, peu après, sous un intitulé percutant, « le pouvoir de tuer »,Politique africaine décrivait d'abord l'extrême violence administrée « sans idéal politique ou idéologique ${ }^{3}$ » par les rebelles révolutionnaires de 1978-1981 dans l'est du Tchad, puis faisait une large place à un témoignage sur les méthodes staliniennes de coercition politique dans la Guinée de Sekou Touré,dont le régime, appartenant à la catégorie des «patrimonialismes sanglants du continent africain », semblait pouvoir être utilement analysé à partir de « l'hypothèse totalitaire ${ }^{4}$. L'attention était également attirée sur l'intérêt qu'il y aurait, pour mieux comprendre « la violence qui afflige les sociétés africaines ${ }^{5}$ ", à ne pas se contenter de tenir pour acquis que, ici plus qu'ailleurs, " l'état de guerre est sous-jacent au politique », ce qui s'explique en grande partie par la traite des esclaves, par « l'ascendance coloniale de bien des violences contemporaines » et par l'exercice de la coercition étatique dans un contexte de mise en dépendance de l'Afrique. Il convenait en effet de considérer aussi que « la violence dont se chargent les relations politiques stricto sensu n'est pas dissociable de celle qui imprègne l'ensemble des rapports sociaux », ce qui porte l'attention sur les « microprocédures» de pouvoir, donc de violence, dont on peut soutenir qu'elles sont enracinées dans la longue durée historique, autrement dit dans la culture ${ }^{6}$.

Ainsi, la piste était ouverte pour l'analyse de la violence enracinée dans la longue durée historique cristallisée dans la culture. Dans cette perspective, J.-F. Bayart évoquait la thèse du philosophe africain Kwasi Wiredu « reliant l'autoritarisme politique à un autoritarisme sociétal récurrent, à un grass roots authoritarianism ", et donnait l'exemple de ces violences ordinaires qui préfigurent et répercutent la brutalité de la compétition politique : les châtiments corporels dont usent et abusent instituteurs ${ }^{7}$, marabouts et parents, ou le déchaînement des affrontements dans le monde occulte où combattent sorciers et « guérisseurs ". À quoi l'on peut ajouter l'extrême violence des foules ameutées contre les petits voleurs, tantôt découpés à la machette, tantôt «braisés » ou brûlés à la soude caustique. Bien sûr, cette violence populaire est aussi liée à celle des conditions de survie au jour le jour (le moindre vol est une catastrophe). Mais on peut néanmoins s'interroger sur l'insensibilité apparente des foules agglutinées autour d'un corps supplicié, surtout quand de jeunes enfants y sont amenés comme à une attraction foraine...

Certes, nul ne peut ignorer «qu'institutionnalisée ou non, [la violence] est la chose la mieux partagée sous toutes les latitudes ». Mais faut-il pour autant, afin d'éviter le piège culturaliste, "dissoudre les phénomènes de violence dans un type d'explication universelle ${ }^{8}$ »? N'y a$\mathrm{t}$-il pas, concurremment avec la violence politique et la violence économique, l'une et l'autre porteuses de violences sociales, des surdéterminations plus souterraines qui, ancrées dans la

\footnotetext{
3 3. P. Doornbos, «La révolution dérapée. La violence dans l’est du Tchad (1978-1981) », Politique Africaine, $\mathrm{n}^{\circ} 7$, septembre 1982, pp. 5-13.

${ }^{4}$ 4. J.-F. Bayart, «L'aveu sous les tropiques », ibid., p. 16.

${ }^{5}$ 5. J.-F. Bayart, « Microprocédures », ibid., pp. 40-42.

6 6. Car « une culture est historique avant d'être culturelle », en ce sens qu'elle n'est « pas un réservoir de représentations constantes [...], mais réactualisation permanente de ces représentations dans le contexte d'une situation historique donnée ». J.-F. Bayart, « Le politique par le bas en Afrique noire...», art. cit., p. 57.

7 7. Dans le même numéro, C.-M. Toulabor observait qu'au Togo la violence à l'école, avec l'usage coutumier des châtiments corporels par le maître, appelait une violence de « résistance » de la part des élèves, mimétique en quelque sorte, puisque pouvant "épouser les formes de la violence qui l'a engendrée » (avec embuscades, passages à tabac...). Mais « plus couramment pratiquée » est la violence occulte de la sorcellerie qui contraint le maître, lui aussi pris dans ce « common knowledge » culturel, à se « blinder » à son tour de protections magiques. Voir « La violence à l'école : le cas d'un village au Togo ", Politique africaine, $\mathrm{n}^{\circ}$ 7, septembre 1982, pp. 43-49.

8 8. J.-F. Bayart, « Microprocédures », art. cit., p. 40.
} 
longue durée des fondements culturels des rapports sociaux et des habitus, seraient le terreau, la toile de fond des violences sociales et politiques ? Ainsi en irait-il des croyances et des pratiques relatives à la sorcellerie dont la violence mortifère est bien réelle, on l'oublie trop souvent, et dont on ne peut guère contester qu'il s'agisse d'un « trait culturel » par excellence, puisqu'elle ne semble pas avoir de cause exogène repérable et que, au contraire, elle constitue l'un des schèmes a priori d'une interprétation rationnellement déductive du monde: ainsi a-telle permis l'appropriation cognitive du phénomène de la traite des esclaves (au Cameroun, la sorcellerie ekong, avec l'achat et la vente de personnes envoûtées, utilisées comme maind'oeuvre servile dans des plantations invisibles, serait une variante de la sorcellerie originaire de la dévoration magique) et de la domination coloniale (la "sorcellerie » blanche, plus puissante que la «sorcellerie » noire), comme, aujourd'hui, elle explique aussi bien les infortunes ou les réussites des gens ordinaires que l'ascension fulgurante d'un entrepreneur politique ou sa chute brutale devant un rival mieux armé en forces occultes.

Si l'on considère ainsi les formes normales, usuelles et banales d'un « autoritarisme sociétal » qui ordonne les relations entre générations successives, entre aînés et cadets et entre hommes et femmes, et si l'on considère que l'un des attributs du pouvoir, à tous les niveaux, tient à l'exercice d'une violence coercitive (en ce qu'elle ne requiert pas l'adhésion explicite des dominés, mais au besoin les contraint, que ce soit par l'usage de la force brute ou par l'usage des pouvoirs occultes, qui sont littéralement des « pouvoirs de tuer » dont les dominants sont censés détenir les formes les plus puissantes), si l'on considère tout cela, en sachant que « là où il y a du pouvoir, il y a de la résistance ", donc de la violence du pouvoir pour vaincre ces résistances et de la violence résistante contre cette violence du pouvoir, on peut comprendre que les sociétés africaines soient consubstantiellement des sociétés violentes, en permanence animées par des rapports de force sous-jacents aux hiérarchies instituées, et que cela soit fidèlement « traduit » dans l'idiome spécifique de la sorcellerie.

\section{Violences enracinées : violences matricielles}

On aurait là, concurremment avec les violences économiques et politiques d'ordre universel, une causalité d'ordre culturel plus spécifique, dont Alain Marie se demande si elle ne tient pas, en dernière analyse, au caractère communautaire des sociétés africaines, celles-ci étant fondées sur une violence à la fois originaire et intrinsèque faite aux individus, et tout particulièrement à ceux qui se trouvent en position de faiblesse sociale, les femmes, les jeunes gens et les cadets. Or, cette violence d'institution ne peut que fabriquer de la violence intérieure, réactive, ressentimentale, toujours prête à faire retour (le «retour du refoulé ») de manière éruptive et brutale (non régulée), sitôt que les circonstances s'y prêtent ou y inclinent ${ }^{9}$.

Ainsi, une micropolitique de la violence pourrait s'inspirer de l'idée particulièrement stimulante selon laquelle « la politique, c'est aussi la relation, conflictuelle souvent, entre l'individuation de la personne et sa socialisation ${ }^{10}$ ". Dès lors, les structures familiales, telles qu'elles

\footnotetext{
${ }^{9}$ Ainsi est-il possible d'expliquer pour partie les atrocités et les massacres de la guerre civile au Liberia par une « ethnopolitique de la violence » faisant un détour par les « guerres lignagères » d'antan, cette ethnopolitique de la violence étant elle-même rapportée au « champ social d'une "violence ordinaire", au miroir de laquelle s'inscrit la violence déréglée et perverse des combattants ", étant entendu qu'il faut simultanément prendre en compte la domination néocoloniale et la « culture de la pauvreté » (alcoolisme, machisme, culte de la jouissance immédiate) si l'on veut comprendre pourquoi la violence sociétale ordinaire d'aujourd'hui «n'attend elle-même qu'une occasion d'instabilité au niveau du pouvoir central pour se manifester dans l'ordre politique». Voir M. Galy, « Liberia, machine perverse. Anthropologie politique du conflit libérien », Cahiers d'études africaines, « Disciplines et déchirures. Les formes de la violence », 150152, XXXVIII (2-4), 1998, pp. 533-553.

$10 \mathrm{~J}$. Pouillon, « De chacun à tout autre, et réciproquement », L’Homme, n 97-98, janvier-juin 1986, pp. 27-38.
} 
sont mises en pratiques (et en représentations) dans l'espace domestique, lieu de la vie quotidienne dans sa banalité la plus ordinaire et lieu privilégié de la socialisation, sont d'emblée appréhendées comme champ où se déploient ces microdispositifs et microprocédures du pouvoir - donc de la violence intrinsèque à tout rapport de pouvoir - qui façonnent les habitus, ces systèmes de prédispositions structurées structurantes déterminant des styles de conduites transposables dans d'autres champs sociaux (celui du politique stricto sensu et de ses violences spécifiques notamment) ${ }^{11}$.

Mais encore faudrait-il expliquer pourquoi l'espace domestique est ainsi structuré autour d'une violence si essentielle qu'il ne peut que l'inculquer comme schème $a$ priori. À partir du paradigme ivoirien, A. Marie montre que les sociétés de type communautaire, parce qu'elles sont d'abord des « unités de survie » dépendant de la solidarité indéfectible de leurs membres, sont nécessairement structurées selon une « logique totalitaire » de « l'antiindividualisme » (comme le dévoilent, entre autres, représentations et pratiques relatives à la sorcellerie) et que, à ce titre, elles refoulent et répriment cette dimension pulsionnelle, universelle de la personne, tout en ne parvenant jamais à l'éradiquer. Dès lors, il n'est pas étonnant que les soupçons, les supputations inquiètes, les procès d'intention, les projections agressives, les récriminations mutuelles et les accusations réversibles, souvent exprimées dans l'idiome de la sorcellerie, mais également les abus de pouvoir puissent, comme le montre Pierre Janin à partir de deux exemples (dans le Burkina rural et en milieu citadin au Bénin), marquer la vie quotidienne au sein de la famille élargie. D'autant plus qu'il s'agit d'un microcosme où règnent en réalité des rapports extrêmement hiérarchiques (entre hommes et femmes, aînés et cadets, parents et enfants), que ces rapports s'y combinent avec une affectivité intense liée à la promiscuité et à l'interdépendance et que, dialectiquement, l'inégalité grandissante des statuts socio-économiques ainsi que les tentatives d'individualisation font aussi de ce microcosme la « caisse de résonance des violences extérieures », celles de la précarité accrue des conditions d'existence et de la raréfaction des ressources. La violence est donc omniprésente dans le huis clos familial; elle hante les rapports sociaux les plus ordinaires et, surtout quand s'épuisent les modalités classiques de régulation des tensions et des conflits, elle s'exerce, parfois au bout d'une longue période d'incubation, au détriment des plus vulnérables pris comme « boucs émissaires » (particulièrement les femmes).

On déboucherait alors sur le constat d'une circularité de la violence, depuis les formes les plus banales, les plus ordinaires, les plus familières et les plus feutrées, en somme les plus enracinées (dans la longue durée historique accoucheuse des logiques structurales qui sont le noyau dur des sociétés et des cultures), jusqu'aux formes les plus extrêmes intervenant dans les situations de crise économique aggravée ou de crise politique à relents de guerre civile pour le contrôle du pouvoir d'État.

Cet enracinement de la violence dans la longue durée est clairement argumenté dans la contribution de Claude Arditi sur le Tchad. Pour comprendre, en effet, les violences ordinaires qui opposent aujourd'hui les nordistes (arabes et musulmans, éleveurs ou commerçants) aux sudistes (agriculteurs polythéistes ou chrétiens), il faut prendre en compte l'ancienne traite transsaharienne des esclaves noirs par les Arabes et les dominations étatiques ou les razzias nordistes sur les sociétés segmentaires du Sud durant la période précoloniale. Mais il faut aussi considérer, à

\footnotetext{
11 D'un article consacré à la violence politique dans l'Algérie contemporaine, on tirera ainsi cette remarque suggestive sur la violence domestique comme mode de socialisation prédisposant les individus à transposer cette violence matricielle dans les rapports sociaux en général : "Très tôt, dans l'espace domestique déjà, l'individu découvre la violence comme mode de socialisation. Le châtiment corporel fait partie du processus normal de l'éducation. Dans le même temps, cette brutalité est érigée en privilège distinctif. Les frères peuvent l'exercer sur les soeurs, les aînés sur les cadets, et le père sur tous. [Ainsi] elle devient une valeur qui épouse parfaitement les contours de la hiérarchie symbolique de la famille comme première matrice du lien social. » A. Moussaoui, « La violence en Algérie. Des crimes et des châtiments », Cahiers d'études africaines, op. cit., pp. 245-269.
} 
partir de la colonisation, le renversement de la domination au profit des agriculteurs sudistes du « Tchad utile » (qui ont trouvé, dans l'école moderne et la maîtrise du français, un moyen de conquérir des positions dans l'État) puis, dans la période postcoloniale, les guerres « civiles » pour le pouvoir entre sudistes et nordistes. Aujourd'hui, les conflits fonciers entre agriculteurs et éleveurs dans le Sud, comme les violences scolaires à N'Djamena (opposant enseignants sudistes aux élèves nordistes et leurs parents), constituent le dernier avatar micropolitique d'une violence multiforme très anciennement enracinée, omniprésente dans les identités, dans les représentations en miroir et dans les parlers populaires, qui sert de toile de fond et de terreau à des violences politiques susceptibles de déboucher sur une nouvelle guerre civile et même sur la partition du pays.

De manière analogue, si, dans les bidonvilles de Nairobi, « la guerre des loyers » décrite par Marie-Ange Goux s'explique par la crise économique, le prix trop élevé des logements dans la ville moderne, la spéculation foncière et immobilière dans les zones d'habitat précaire et la migration massive des paysans chassés des campagnes vers les bidonvilles par la misère, les formes violentes que prend cette lutte appellent d'autres explications. En effet, les affronte-ments ont d'abord opposé des bandes armées de locataires luo à des bandes de propriétaires anciens d'origine nubienne, puis se sont étendus à d'autres bidonvilles, mettant aux prises milices armées recrutées par de gros propriétaires spéculateurs kikuyu liés aux politiciens et groupes d'autodéfense luo, et ce sur fond de guerres des gangs, de luttes électorales (les politiciens finissant par chercher le soutien des locataires) et de renversements d'alliance entre partis poli-tiques. Cependant, les regroupements ethniques au sein des bidonvilles et le fait que la jeunesse, tout particulièrement luo, soit exclue des processus d'appropriation foncière, transforment ce qui pourrait être une « lutte de classes » en une «guerre des générations » (jeunes locataires contre vieux propriétaires, cadets contre aînés) surdéterminée par des tensions ethniques et des rivalités politiques.

Enfin, dans l'ancien township de Soweto, la démocratisation de l'après apartheid se trouve paradoxalement instrumentalisée et même détournée par une guerre des sexes inédite. En effet, une loi récente sur la violence domestique, en élargissant le champ des délits et en faisant obligation à la police d'agir sitôt la plainte enregistrée, a considérablement multiplié le nombre des plaintes déposées par les femmes contre leur conjoint ou compagnon. Mais ces plaintes sont retirées avant les poursuites judiciaires : ainsi les femmes utilisent-elles les policiers comme des travailleurs sociaux dans la gestion de leurs relations de couple afin de lutter contre les brutalités de leurs partenaires sans rompre pour autant la relation. Cette «privatisation de la police " et le recours à l'État dans les violences familiales et conjugales ordinaires sont l'expression d'un besoin d'État en tant qu'instance s'arrogeant le monopole de la violence et en dépossédant les personnes privées, ce qui peut s'interpréter comme une demande de « civilisation » des rapports sociaux ordinaires et coutumiers, marqués par une violence diffuse et systématique. Certes, des conditions d'existence extrêmement précaires (misère, alcoolisme, promiscuité, chômage, trafics et usage de drogue, guerres des gangs, jalousies liées à la pénurie...) expliquent largement cette demande d'État, mais il n'est pas interdit de penser qu'elle relève plus généralement, de la part des femmes, d'un désir de réforme radicale des relations entre les genres, caractérisées par une violence enracinée dans les coutumes et dans les habitus.

Certes, la piste de la violence ordinaire, enracinée dans la longue durée, donc dans la culture, se ramifie à l'envi tant le domaine, malgré les invites initiales précédemment rappelées, est divers et complexe mais resté peu fréquenté. Les textes du présent dossier sont donc eux aussi un appel à poursuivre plus avant. Car les violences extrêmes, celles qui se manifestent ostensiblement et cruellement dans le champ de la lutte pour le pouvoir et les richesses, ont continué d'attirer davantage l'attention des chercheurs. Même Politique africaine 
n'a guère frayé la piste de "l'économie de la violence » sous ses « formes routinières qui ne sont pas directement ni fortement articulées sur la compétition politique ${ }^{12} »$. L'exemple du triple numéro des Cahiers d'études africaines récemment consacré aux « formes de la violence » est également révélateur : sur une vingtaine d'articles, les trois quarts traitent des violences politicoguerrières ou de la délinquance et les autres n'abordent la « violence sociétale ordinaire » que comme phénomène fournissant une explication d'appoint à la violence sociale de la misère et de l'inégalité et à la violence politique des luttes armées pour le pouvoir - c'est-à-dire, dans les deux cas, à la violence comme manifestation de situations de crises graves au niveau macrosocial. Pourtant, comme le fait observer l'un des contributeurs de ces Cahiers, « par certains aspects, la violence peut constituer un principe organisateur de la société, pas seulement dans les moments de crise, mais aussi comme élément structurant d'une partie des valeurs et de l'expérience humaines ». En bref, on peut regretter que les « études de sciences sociales qui traitent systématiquement de l'interaction violente comme une dimension fondamentale de la (re)production de la socialité humaine soient toujours relativement rares ${ }^{13}$ ".

À condition d'éviter les deux écueils du culturalisme (et de sa variante essentialiste) et du déterminisme économique, le thème des violences enracinées dans l'ordinaire des rapports sociaux fondamentaux et des représentations spontanées devrait donc appeler d'autres recherches où se croiseraient utilement les points de vue de l'anthropologie, de la science politique, de la sociologie et de l'ethnopsychanalyse. Certaines apories des violences contemporaines les plus inhumaines et les plus rebelles à l'analyse rationnelle s'en trouveraient sans doute mieux abordées.

Pierre Janin, IRD-Ouagadougou, Université Paris-I (IEDES), et Alain Marie, université Paris-I (IEDES)

\footnotetext{
12 12. R. Marchal, «Surveillance et répression en postcolonie », Politique africaine, dossier «Violence et pouvoir », $n^{\circ} 42$, juin 1991, pp. 40-50.

13 13. J. Abbink, « Ritual and political forms of violent practices among the Suri of Southern Ethiopia », Cahiers d'études africaines, op. cit., pp. 271-295.
} 